\title{
The effect of moderate acute psychological stress on working memory-related neural activity is modulated by a genetic variation in catecholaminergic function in humans
}

\author{
Shaozheng Qin 1,2*, Helena Cousijn 1,3, Mark Rijpkema ${ }^{1}$, Jing Luo ${ }^{4,5}$, Barbara Franke ${ }^{1,6}$, \\ Erno J. Hermans ${ }^{1,7}$ and Guillén Fernández ${ }^{1,7}$ \\ Donders Institute for Brain, Cognition and Behaviour, Radboud University Nijmegen Medical Centre, Nijmegen, Netherlands \\ ${ }^{2}$ Department of Psychiatry and Behavioral Sciences, Stanford University School of Medicine, Stanford, CA, USA \\ ${ }^{3}$ Department of Psychiatry, Warneford Hospital, University of Oxford, Oxford, UK \\ ${ }^{4}$ Key Laboratory of Mental Health, Institute of Psychology, Chinese Academy of Sciences, Beijing, China \\ ${ }^{5}$ Beijing Key Laboratory of Learning and Cognition, Department of Psychology, Capital Normal University, Beijing, China \\ ${ }^{6}$ Department of Human Genetics, Radboud University Nijmegen Medical Centre, Nijmegen, Netherlands \\ Department for Cognitive Neuroscience, Radboud University Nijmegen Medical Centre, Nijmegen, Netherlands
}

\section{Edited by:}

Florin Dolcos, University of Illinois Urbana-Champaign, USA

Reviewed by:

Barry Setlow, University of Florida, USA

Antonio Pereira, Federal University of Rio Grande do Norte, Brazil Nicole Oei, Leiden University Medical Center, Netherlands

\section{*Correspondence:}

Shaozheng Qin, Department of Psychiatry and Behavioral Sciences, Stanford University School of Medicine, 401 Quarry Rd, Stanford, CA 94305-5719, USA.

e-mail:szqin@stanford.edu
Acute stress has an important impact on higher-order cognitive functions supported by the prefrontal cortex (PFC) such as working memory (WM). In rodents, such effects are mediated by stress-induced alterations in catecholaminergic signaling, but human data in support of this notion is lacking. A common variation in the gene encoding Catechol-O-methyltransferase (COMT) is known to affect basal catecholaminergic availability and PFC functions. Here, we investigated whether this genetic variation (Val158Met) modulates effects of stress on WM-related neural activity in humans. In a counterbalanced crossover design, 41 healthy young men underwent functional magnetic resonance imaging (fMRI) while performing a numerical N-back WM task embedded in a stressful or neutral context. Moderate psychological stress was induced by a well-controlled procedure involving viewing strongly aversive (versus emotionally neutral) movie material in combination with a self-referencing instruction. Acute stress resulted in genotype-dependent effects on WM performance and WM-related activation in the dorsolateral PFC, with a relatively negative impact of stress in COMT Met-homozygotes as opposed to a relatively positive effect in Val-carriers. A parallel interaction was found for WM-related deactivation in the anterior medial temporal lobe (MTL). Our findings suggest that individuals with higher baseline catecholaminergic availability (COMT Met-homozygotes) appear to reach a supraoptimal state under moderate levels of stress. In contrast, individuals with lower baselines (Val-carriers) may reach an optimal state. Thus, our data show that effects of acute stress on higher-order cognitive functions vary depending on catecholaminergic availability at baseline, and thereby corroborate animal models of catecholaminergic signaling that propose a non-linear relationship between catecholaminergic activity and prefrontal functions.

Keywords: stress, working memory, Catechol-O-methyltransferase, catecholamine, prefrontal cortex, fMR

\section{INTRODUCTION}

Acute stress has an important impact on higher-order cognitive function such as working memory (WM). These effects are believed to result from stress-induced alterations of large-scale brain network functioning including the prefrontal cortex (PFC), through stress-sensitive neuromodulatory systems (GoldmanRakic, 1995; Aston-Jones and Cohen, 2005; Arnsten, 2007, 2009; McEwen, 2007; Sara, 2009; Hermans et al., 2011). In particular, stress-induced changes in catecholaminergic signaling play a central role in modulating prefrontal functions in rodents (Arnsten and Goldman-Rakic, 1998; Arnsten, 2009). Yet, direct human data in support of this notion is lacking. A variation (Val158Met) in the gene encoding Catechol-O-methyltransferase (COMT) is associated with individual differences in basal catecholaminergic availability (Lotta et al., 1995; Mannisto and Kaakkola, 1999) and prefrontal functions (Chen et al., 2004). We, therefore, conjecture that effects of acute stress may interact with COMT genotype to alter WM-related PFC function in humans.

Exposure to acute stress leads to rapid activation of sympathetic nervous system (SNS) and hypothalamic-pituitary-adrenal (HPA) axis, and is accompanied by a tonical increase of central release of catecholamine levels (de Kloet et al., 2005; Arnsten, 
2009; Joels and Baram, 2009). Through ascending projections from brainstem nuclei where the locus coeruleus norepinephrine and midbrain dopaminergic systems locate, these stress-sensitive catecholamines alter neuronal functioning of widely distributed brain regions, particularly including the PFC (Arnsten and Li, 2005; Aston-Jones and Cohen, 2005; Arnsten, 2009; Sara, 2009). Converging evidence from human behavioral and neuroimaging studies have confirmed that acute stress indeed alters WM performance with both detrimental (Elzinga and Roelofs, 2005; Oei et al., 2006; Luethi et al., 2008; Schoofs et al., 2008) and enhancing (Lewis et al., 2008; Weerda et al., 2010; Hidalgo et al., 2011) effects, likely through altered efficiency of WM-related processing in dorsolateral PFC (Porcelli et al., 2008; Qin et al., 2009; Weerda et al., 2010). Interestingly, animal studies suggest that stress-sensitive catecholamines exert an inverted U-shaped influence on prefrontal functions (Aston-Jones and Cohen, 2005; Vijayraghavan et al., 2007), in which prefrontal functioning reaches an optimum at an intermediate level of catecholaminergic activity (Arnsten and Li, 2005; Aston-Jones and Cohen, 2005; Vijayraghavan et al., 2007). These implicate that acute stress may have variable (i.e., either detrimental or enhancing) effects on PFC functions depending on levels of stress-induced catecholaminergic activity.

The intracellular enzyme COMT plays a key role in regulating catecholaminergic availability. A single nucleotide polymorphism (SNP) in the gene coding COMT, which causes a Valine-toMethionine substitution at codon 158 (Val158Met), leads to a 3-4 fold decrease in the ability of this enzyme to catabolize catecholamines (Lotta et al., 1995; Mannisto and Kaakkola, 1999). As a consequence, basal levels of catecholaminergic availability in Met-homozygotes are presumed to be increased throughout the cortex, particularly for the PFC (Chen et al., 2004). Associations have been found in humans between COMT genotype and higher-order PFC functions and emotional processing in both health and disease (Drabant et al., 2006; Meyer-Lindenberg et al., 2006; Bishop et al., 2008). Interestingly, recent evidence from human and rodent studies suggests that COMT genotype may interact with catecholaminergic manipulations altering PFC function (Mattay et al., 2003; Bertolino et al., 2004; Papaleo et al., 2008). Specifically, one study demonstrated that COMT knockout mice (Met-like genotype) is associated with better WM functioning at baseline, but also with a higher sensitivity to stress. In contrast, the COMT-Val transgenic mice (Val-like genotype) are associated with inferior WM performance at baseline, but are less sensitive to stress (Papaleo et al., 2008). Based on these findings and the aforementioned inverted $U$-shaped relationship between catecholaminergic signaling and neurocognitive functioning, one may hypothesize that effects of experimentally induced moderate stress on WM-related prefrontal activity in humans would exhibit a similar genotype-dependency, with stronger detrimental effects of stress in COMT Met-homozygotes.

To test this hypothesis, 41 healthy young men underwent functional magnetic resonance imaging (fMRI) while performing a numerical N-back WM task (including 2- and 0 -back conditions). In counterbalanced order, one session involved stress induction while the other session served as control. Moderate psychological stress was induced experimentally by a standardized cinematographic procedure in which participants saw strongly aversive (vs. neutral control) movie material combined with a self-referencing instruction, which has been shown previously to elicit physiological and psychological stress responses (Henckens et al., 2009; Qin et al., 2009; Hermans et al., 2011). To validate our stress manipulation, measures of heart rate (HR), $\alpha$-amylase, salivary cortisol, and subjective negative affect were acquired prior to, during and after fMRI scanning. Given the relatively low frequency of Val-homozygotes in our sample, Val-carriers were treated as a single group in all analyses. We predicted that moderate psychological stress would have an interactive effect on the differential neural activity reflecting WM load (2- vs. 0-back) in Met-homozygotes and Val-carriers, with a stronger stress-induced reduction of differential dorsolateral PFC activity in Met-homozygotes. Because WM-related activation of the dorsolateral PFC is normally accompanied by deactivation in the medial temporal lobe (MTL), and adaptive changes in reciprocal organization of dorsal executive and ventral affective brain areas under arousal and stressful circumstance (Dolcos and McCarthy, 2006; Oei et al., 2011; Cousijn et al., 2012), we also conjectured a corresponding genotype-dependent effect in the MTL.

\section{MATERIALS AND METHODS PARTICIPANTS}

Forty-one young, healthy, right-handed males (Caucasian ethnicity, aged 18-35 years) with normal or corrected-to-normal vision participated in this study. There are 16 Met-homozygotes (Met/Met), 7 Val-homozygotes (Val/Val) and 18 heterozygotes (Val/Met). Participants reported no history of neurological, psychiatric, or endocrine disease, and no current use of psychoactive drugs or corticosteroids. None of them had experienced severe physical or emotional trauma. Only men were included to avoid confounds related to gender differences and menstrual cycle-dependent variance in stress responsiveness (Kirschbaum et al., 1999; Kudielka and Kirschbaum, 2005). All had participated before in MRI experiments to minimize stress responses evoked by unfamiliarity with the environment and procedures. The study was in accordance with the Declaration of Helsinki and institutional guidelines of the local ethics committee (CMO Region Arnhem-Nijmegen, Netherlands). All participants gave written informed consent. Data from two participants (1 Methomozygote and 1 heterozygote) were excluded from further analysis completely, either due to technical failure or excessive head movement during scanning.

Participants were tested in a mixed factorial design with stress induction as within-subject factor and genotype as betweensubject factor. The groups (COMT Met-homozygotes and Valcarriers) did not differ significantly regarding age $(p>0.1)$ and trait anxiety $(p>0.4)$ (see Table $\mathbf{1})$.

\section{GENERAL PROCEDURE}

The experiment was carried out in the afternoon (after $1 \mathrm{pm}$ ) to ensure relatively stable and low levels of endogenous cortisol. After arrival, $1 \mathrm{~h}$ before scanning, participants trained on the WM task extensively and completed various questionnaires. To determine whether baseline cortisol levels differed between COMT 
Table 1 | Participant demographics.

\begin{tabular}{llll}
\hline & Met-homozygotes $(\boldsymbol{n}=\mathbf{1 5})$ & Val-carriers $(\boldsymbol{n}=\mathbf{2 4})$ & Total $(\boldsymbol{n}=\mathbf{3 9})$ \\
\hline Age $($ mean \pm SE) & $23.51 \pm 1.13$ & $23.82 \pm 5.57$ & $23.67 \pm 5.55$ \\
Anxiety $($ mean \pm SE) & $28.52 \pm 5.57$ & $31.00 \pm 5.33$ & $30.49 \pm 5.50$ \\
\hline
\end{tabular}

Note: Due to either technical failure or excessive head movement during scanning, data from two participants were excluded from further analyses. SE, standard of error of mean; anxiety, trait anxiety scores.

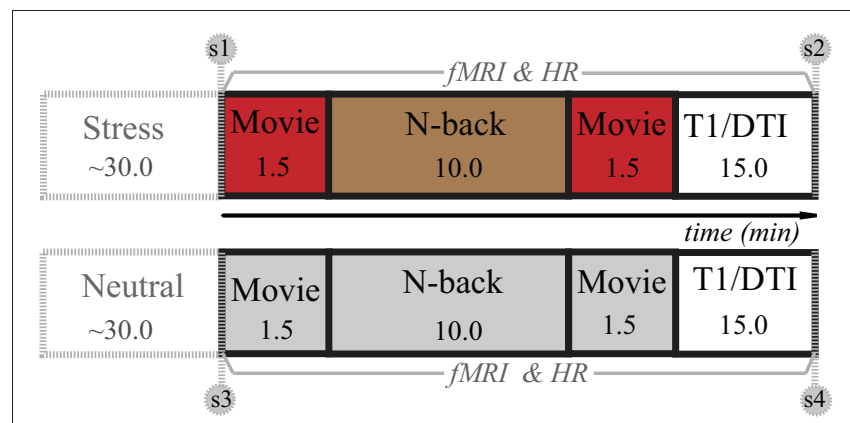

FIGURE 1 | Overview of experimental design. The experiment consisted of stress and control conditions (or sessions), and the order was counterbalanced across participants. After preparation outside the scanner, a numeric N-back WM task was administered twice: in one session it was embedded in a stressful context and the other one in a neutral control condition (see Stress induction for details). Note: The numbers in the squares indicate the time in minutes. S1-S4 represents four saliva samples coinciding with subjective mood ratings (PANAS). T1 and DTI stand for a high-resolution anatomical scan and a diffusion tensor imaging scan.

genotypes, all participants were asked to take one saliva sample as baseline in the late afternoon on the day before the experiment at home. Other saliva samples and subjective affect positive and negative affect scales (PANAS) were obtained before and after both experimental conditions. The actual fMRI experiment consisted of four short movie clips to ensure that tasks of interest were fully embedded in a continuously stressful (or neutral control) context. Between the first and second movie clips, participants performed the numeric N-back task (Figure 1). Before and after the stress and control condition, saliva samples and subjective affect ratings were collected and HR was measured throughout the experiment. The two conditions were separated by approximately $20 \mathrm{~min}$, consisting of a structural MRI scan and a perfusion fMRI scan.

\section{STRESS INDUCTION}

In the stress induction condition, moderate acute psychological stress was induced by showing short movie clips within the MRI scanner containing scenes with strongly aversive content (extreme violence) selected from a commercially available movie (Irreversible, 2002, by Gaspar Noé). The N-back task was fully embedded in short movie clips (Figure 1) containing scenes with extremely aversive content. In the control condition, participants watched equally long movie clips from another movie (Comment j'ai tué mon père, 2001, by Anne Fontaine) which were equal in luminance and similar in language but contained only nonarousing scenes. Matching for audiovisual characteristics was performed by the authors by selecting aversive and neutral clips out of a set of candidate clips which best matched on the measures described in Hermans et al., 2011. After short introductory texts, participants were asked to watch the movies attentively and take an eyewitness perspective as to involve them maximally in the action taking place in the movie clips. The WM task was closely surrounded by two movie clips in time in order to boost stress induction and create a continuously stressful context.

We chose to use a stress induction procedure involving exposure to aversive cinematographic material in combination with self-referencing instruction, because of the following considerations (also see Hermans et al., 2011). First, this procedure allows us to create an ecologically valid model for examining stress-induced adaptive alterations (i.e., hypersensitivity at the cost of higher-order cognitive functions) when exposing to acutely stressful experiences in the real world that may ultimately trigger emotional trauma with detrimental effects on various higher-order prefrontal cognitive functions. Second, exposure to highly aversive films in combination with selfreferencing instruction meets the requirements for the physiological stress response in humans, particularly with activation of the sympatho-adrenomedullary system (SAM) and substantial elevation of epinephrine and norepinephrine levels, as described by Mason (Mason, 1968). Finally, unlike stressors based on public speaking, and cognitive performance in combination with negative social evaluation (Kirschbaum et al., 1993; Wang et al., 2005; Pruessner et al., 2008), our stress induction is likely to yield emotion focused rather than social or problem-focused coping strategies and plausibly triggers a state of fearful arousal. From a more general and broader standpoint of view, such a state of fearful arousal has been long believed to be an important feature of psychological stress in both animals and humans (Mason, 1968; Arnsten and Goldman-Rakic, 1998; Mobbs et al., 2007; Arnsten, 2009; Shackman et al., 2011). Moreover, previous studies have shown that this method elicits a measurable psychological, physiological, and neuroendocrine stress response (Henckens et al., 2009; Qin et al., 2009; van Marle et al., 2009), although it has relatively lower cortisol elevations than other social and/or cognitive challenging stressors. Notably, our stress induction procedure has been validated by one recent pharmacological study in humans, demonstrating that stress-sensitive noradrenergic (probably dopaminergic as well) activity plays a central role in regulating acute psychological stress (Hermans et al., 2011). This nicely fits the primary goal of our present study addressing how a genetic variation in catecholaminergic function modulates the effect of acute psychological stress on WM processing in humans.

\section{N-BACK TASK}

Using a blocked-design, participants completed six cycles of alternating 0 - and 2-back conditions interleaved by a jittered 
resting-fixation baseline ranging from 8 to $12 \mathrm{~s}$. Within each block, a random digit sequence consisting of 15 single digits was shown to participants. Each digit was presented for $400 \mathrm{~ms}$, followed by an inter-stimulus interval of $1400 \mathrm{~ms}$. Each block lasted 27 s. During the 0-back condition, participants were asked to detect whether the current item on the screen was a " 1 " or not. During the 2-back condition, participants were asked to detect whether the current item had appeared two positions back in the sequence. Participants were instructed to make a button press with their index finger when detecting a target. Before fMRI scanning, they were extensively trained in performing the task to minimize interindividual variability and reduce practice effects. During this pretraining session, we trained every participant to perform 10 cycles of alternating 0 - and 2-back conditions as used in our previous study (Qin et al., 2009). We did not set up specific performance criteria during pretraining, because we intended to minimize potential anticipatory and psychological stress induction when performing challenging WM task conjoint with performance evaluation (Dickerson and Kemeny, 2004; Pruessner et al., 2008).

\section{PHYSIOLOGICAL AND PSYCHOLOGICAL MEASUREMENTS OF STRESS}

To assess the SNS and HPA axis responses to the stress manipulation, saliva was sampled with salivette collection devices to determine the levels of $\alpha$-amylase and cortisol. Samples were taken on the day before the experiment and before and after both conditions (five in total) and were stored at $-20^{\circ} \mathrm{C}$ until analysis. The analysis (Cousijn et al., 2010; Strahler et al., 2010) was carried out at the Biopsychology Department in Dresden, where samples were prepared for biochemical analysis by centrifuging at $1500 \times g$ for $5 \mathrm{~min}$, which resulted in a clear supernatant of low viscosity. Salivary-free cortisol concentrations were determined with a chemiluminescence assay with high sensitivity of $0.16 \mathrm{ng} / \mathrm{mL}$ (IBL). Concentration of $\alpha$-amylase in saliva was measured by an enzyme kinetic method: saliva was processed on a Genesis RSP8/150 liquid handling system (Tecan). First, saliva was diluted 1:625 with double-distilled water by the liquid handling system. Twenty microliters of diluted saliva and standard were then transferred into standard transparent 96-well microplates (Roth). Standard was prepared from "Calibrator f.a.s." solution (Roche Diagnostics) with concentrations of $326,163,81.5,40.75,20.38$, 10.19 , and 5.01 U/L $\alpha$-amylase, respectively, and double-distilled water as zero standard. After that, $80 \mathrm{~mL}$ of substrate reagent ( $\alpha$-amylase EPS Sys; Roche Diagnostics) were pipetted into each well using a multichannel pipette. The microplate containing sample and substrate was then warmed to $37^{\circ} \mathrm{C}$ by incubation in a water bath for $90 \mathrm{~s}$. Immediately afterwards a first interference measurement was obtained at a wavelength of $405 \mathrm{~nm}$ with a standard ELISA reader (Anthos Labtech Instruments HT2). The plate was then incubated for another $5 \mathrm{~min}$ at $37^{\circ} \mathrm{C}$ in the water bath, before a second measurement at $405 \mathrm{~nm}$ was taken. Increases in absorbance were calculated for unknowns and standards. Increases of absorbance of diluted samples were transformed to $\alpha$-amylase concentrations using a linear regression calculated for each microplate (Graphpad Prism 4.0 c for MacOSX; Graphpad Software). For one subject no data were acquired and for one subject the analysis did not succeed, whereas data of a third subject were not taken into account because he consumed caffeine shortly before the experiment. Behavioral and fMRI data quality of these three subjects were good, thus they were included in further analysis.

To assess autonomic activity throughout the experiment, we continuously recorded HR with an infrared pulse oximeter (MRcompatible) placed on the index finger of the left hand. Offline artifact correction and analysis of the HR frequency was done with in-house software. The HR frequency was averaged for the duration of each movie clip and the task. HR data from one participant were excluded due to excessive artifacts, while corresponding behavioral and fMRI data were included into further analysis.

Mood state was assessed using the positive and negative affect scale (PANAS) questionnaire (Watson et al., 1988) at four time points coinciding with the collection of salivary samples.

\section{GENOTYPING}

Genetic analyses were performed at the Department of Human Genetics of the Radboud University Nijmegen Medical Centre, in a laboratory which has a quality certification according to CCKL criteria. High molecular weight DNA was isolated from saliva using Oragene containers (DNA Genotek, Ottawa, ON, Canada) according to the protocol supplied by the manufacturer. All participants were genotyped for the COMT SNP rs4680 ( $\mathrm{G}>\mathrm{A}$; Val1 58 Met) using Taqman ${ }^{\circledR}$ analysis (Applied Biosystems, Nieuwerkerk a/d IJssel, Netherlands). Genotyping was carried out in a volume of $10 \mu \mathrm{l}$ containing $10 \mathrm{ng}$ of genomic DNA, $5 \mu \mathrm{l}$ of Taqman Mastermix (2×; Applied Biosytems), $0.375 \mu \mathrm{l}$ of the Taqman assay, and $3.625 \mu \mathrm{l}$ of Milli-Q. The amplification protocol consisted of an initial denaturation step at $95^{\circ} \mathrm{C}$ for $10 \mathrm{~min}$ followed by 40 cycles of denaturation at $92^{\circ} \mathrm{C}$ for $15 \mathrm{~s}$ and annealing and extension at $60^{\circ} \mathrm{C}$ for $60 \mathrm{~s}$. Allele-specific fluorescence was subsequently measured on an ABI 7500 FAST (Applied Biosystems). Taqman genotyping assays were validated before use and 5\% duplicates and blanks were taken along as quality controls during genotyping. Genotyping results were only considered valid if duplicates and blanks were called correctly and genotypes could be called for at least $95 \%$ of the sample tested. All genotype frequencies were tested for Hardy-Weinberg Equilibrium.

\section{fMRI DATA ACQUISITION}

During MRI scanning, whole brain T2*-weighted echo planar imaging based on blood oxygenation level-dependent contrast (EPI-BOLD) fMRI data were acquired with a Siemens Trio 3.0 T MR-scanner (Erlangen, Germany) using an ascending slice acquisition sequence. Parameters of the sequence are following: 37 axial slices; volume repetition time, TR $2.18 \mathrm{~s}$; echo time, TE $25 \mathrm{~ms} ; 80^{\circ}$ flip angle; slice matrix size, $64 \times 64$; slice-thickness, $3.0 \mathrm{~mm}$; slice gap, $0.3 \mathrm{~mm}$, field of view, FOV $212 \times 212 \mathrm{~mm}$. Two hundred six volumes were acquired during the N-back task. High-resolution structural images $(1 \times 1 \times 1 \mathrm{~mm})$ were acquired using a T1-weighted 3D magnetization-prepared rapid gradientecho (MPRAGE) sequence (TR, $2.3 \mathrm{~s}$, TE, $3.03 \mathrm{~ms}, 8^{\circ}$ flip angle, 192 contiguous sagittal slices, slice matrix size, $256 \times 256$, FOV, $256 \times 256 \mathrm{~mm})$. 
Table 2 | Physiological and psychological measurements of stress $(n=39)$.

\begin{tabular}{|c|c|c|c|c|c|c|c|c|c|}
\hline & \multicolumn{2}{|c|}{ Cortisol (nmol/I) } & \multicolumn{2}{|c|}{$\alpha$-amylase (U/I) } & \multicolumn{3}{|c|}{ HR (beats per minute) } & \multicolumn{2}{|c|}{ Negative affect } \\
\hline $\begin{array}{l}\text { Stress } \\
\text { (mean } \pm \text { SE) }\end{array}$ & $7.68 \pm 0.80$ & $7.97 \pm 0.93$ & $47.77 \pm 6.09$ & $58.06 \pm 7.55$ & $68.10 \pm 1.85$ & $64.71 \pm 1.55$ & $65.80 \pm 1.72$ & $12.80 \pm 0.47$ & $16.28 \pm 0.89$ \\
\hline $\begin{array}{l}\text { Control } \\
\text { (mean } \pm \text { SE) }\end{array}$ & $8.32 \pm 0.73$ & $6.39 \pm 0.46$ & $55.30 \pm 7.14$ & $49.36 \pm 6.71$ & $59.92 \pm 1.32$ & $63.24 \pm 1.42$ & $60.29 \pm 1.34$ & $13.13 \pm 0.61$ & $12.54 \pm 0.46$ \\
\hline
\end{tabular}

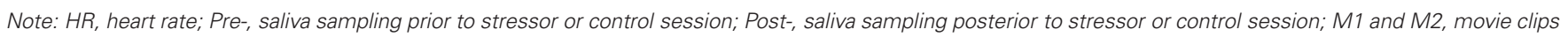
prior and posterior to N-back task; Negative affect, negative affect scale; SE, standard error of mean.

Table 3 | Averaged (mean \pm SE) accuracy and reaction times for 0- and 2-back working memory $(n=39)$.

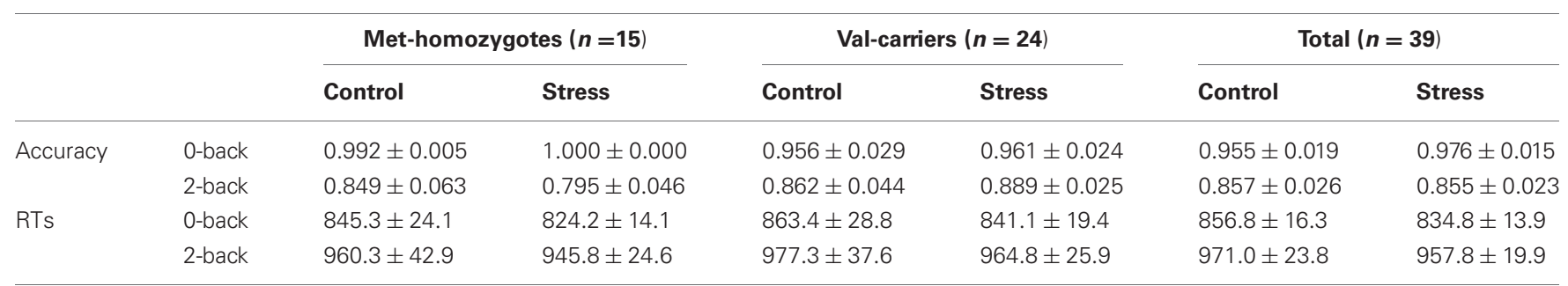

Note: $R T$, reaction times; $S E$, standard error of mean.

\section{fMRI DATA ANALYSIS}

Image preprocessing and statistical analysis was performed using SPM5 (http://www.fil.ion.ucl.ac.uk/spm/). The first five EPI volumes were discarded to allow for T1-equilibration. Remaining functional images were rigid-body motion corrected and the mean image was coregistered to each participant's T1-weighted MR-image. Subsequently, images were transformed into a common stereotactic space, and resampled into $2 \mathrm{~mm}$ isotropic voxels. Finally, images were spatially smoothed by convolving with an isotropic 3D-Gaussian kernel ( $8 \mathrm{~mm}$ full width at half maximum). The data were statistically analyzed using general linear models and statistical parametric mapping (Friston et al., 1995).

To assess neural activity associated with 0 - and 2-back conditions, the two conditions were modeled separately as boxcar regressors and convolved with the canonical hemodynamic response function in SPM5. Additionally, realignment parameters were included to account for movement-related variability. The analysis furthermore included high-pass filtering using a cutoff of $1 / 128 \mathrm{~Hz}$, global intensity normalization, and serial correlations correction using a first-order autoregressive [or $\mathrm{AR}(1)$ ] model.

The contrast parameter images for the 2- vs. the 0-back condition, generated at the single-subject level, were submitted to a second-level analysis within a stress-by-genotype group mixed factorial analysis of variance (ANOVA). The analysis of WM-related deactivations was conducted based on the opposite contrast of 0 - vs. 2-back condition. We used an alpha of 0.05 corrected for multiple comparisons based on suprathreshold cluster size statistics (Worsley et al., 1996). The initial threshold for this analysis was set at $p<0.001$, uncorrected. Given our clear hypotheses regarding the PFC, this region was additionally investigated with a reduced search region consisting of

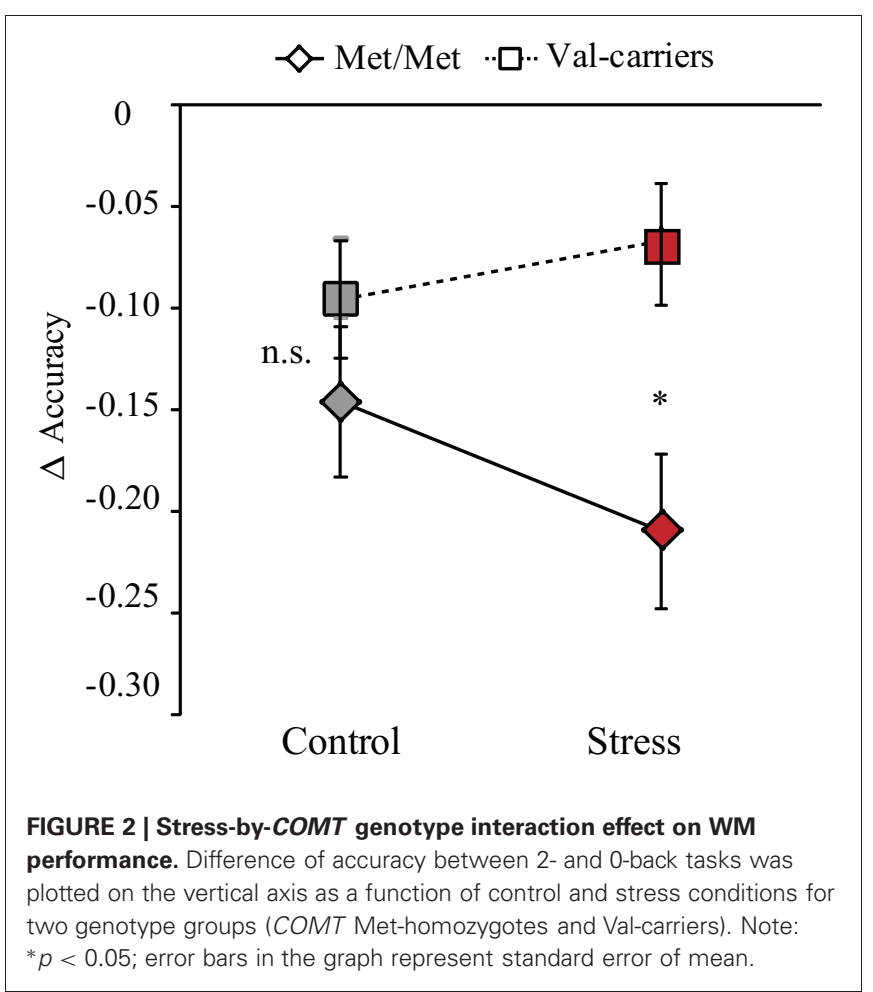

an unbiased, anatomically defined, gray matter mask of the PFC covering the entire prefrontal areas, by using non-stationary suprathreshold cluster-size approach based on Monte-Carlo simulations (Forman et al., 1995; Ward, 2000; Nichols and Hayasaka, 2003; Hayasaka et al., 2004). This approach allows us to determine the minimum cluster size that controls for false positive 
rate. The PFC mask was anatomically defined by combination of a gray matter mask and the anatomical automatic labeling (AAL) template of the PFC. Monte-Carlo simulations were implemented in Matlab using methods similar to the AlphaSim procedure. Ten thousand iterations of random 3D images, with the same resolution and dimensions as the fMRI data, were generated. The resulting images with the gray matter mask of interest were smoothed with the same $8 \mathrm{~mm}$ FWHM Gaussian kernel used to smooth our fMRI data. The maximum cluster size was then computed for each iteration and the probability distribution was estimated across the 10,000 iterations. The cluster threshold corresponding to a voxel-wise threshold of $p<0.001$ for height, and a significance level of $p<0.05$ corrected for multiple spatial comparisons was determined to be 28 voxels. Parameter estimates were extracted from clusters of activation associated with interaction effects to characterize the neural activation patterns of the stress and neutral conditions in the two groups using MarsBar (Brett et al., 2002).

\section{RESULTS}

\section{EFFECTIVENESS OF STRESS INDUCTION}

Measurements of salivary cortisol, $\alpha$-amylase, and subjective negative affect were submitted to separate $2 \times 2 \times 2$ repeated measures ANOVAs with stress (stress induction vs. control) and time (pre vs. post) as within-subject factors, and genotype (COMT
Met-homozygotes vs. Val-carriers) as between-subject factor, and with the order effect of stress manipulation as a covariate of no interest (similarly hereinafter for other ANOVAs including functional imaging data). Time-by-stress interactions were found for salivary cortisol $\left[F_{(1,34)}=4.46, p<0.05\right], \alpha$-amylase $\left[F_{(1,34)}=\right.$ $8.59, p=0.006]$ (Table 2 ), and negative affect $\left[F_{(1,37)}=20.49\right.$, $p<0.001]$. For cortisol, a decrease was observed from preto post-stressor levels in the neutral condition $\left[t_{(35)}=5.12\right.$, $p<0.001]$, but no significant change in the stress condition $\left[t_{(35)}<1\right]$. For $\alpha$-amylase, we observed an increase in the stress condition $\left[t_{(35)}=2.33, p<0.03\right]$ and a decrease in the control condition $\left[t_{(35)}=2.07, p<0.05\right]$. Negative affect increased in the stress condition only $\left[F_{(1,37)}=8.23, p<0.01\right]$. In addition, a two independent samples $T$ test revealed $t$ difference $\left[t_{(34)}<1\right]$ in cortisol between Met-homozygotes (7.89 \pm 7.92$)$ and Val-carriers $(8.04 \pm 2.17)$ at baseline which were collected one day prior to the experiment.

HR data were analyzed with a $2 \times 3 \times 2$ repeated measures ANOVA across averaged measures during the N-back task and the two surrounding movie clips. We found a main effect of stress $\left[F_{(1,74)}=46.62, p<0.001\right]$, and further $t$-tests confirmed that HR was consistently higher during all three phases of the experiment in the stress condition [all $t_{(38)}>2.29, p<0.03$ ].

None of the statistical tests described above revealed significant interaction effects of genotype and order-related effects.

Table 4 | Brain activations related to WM, and modulations of stress and COMT genotype.

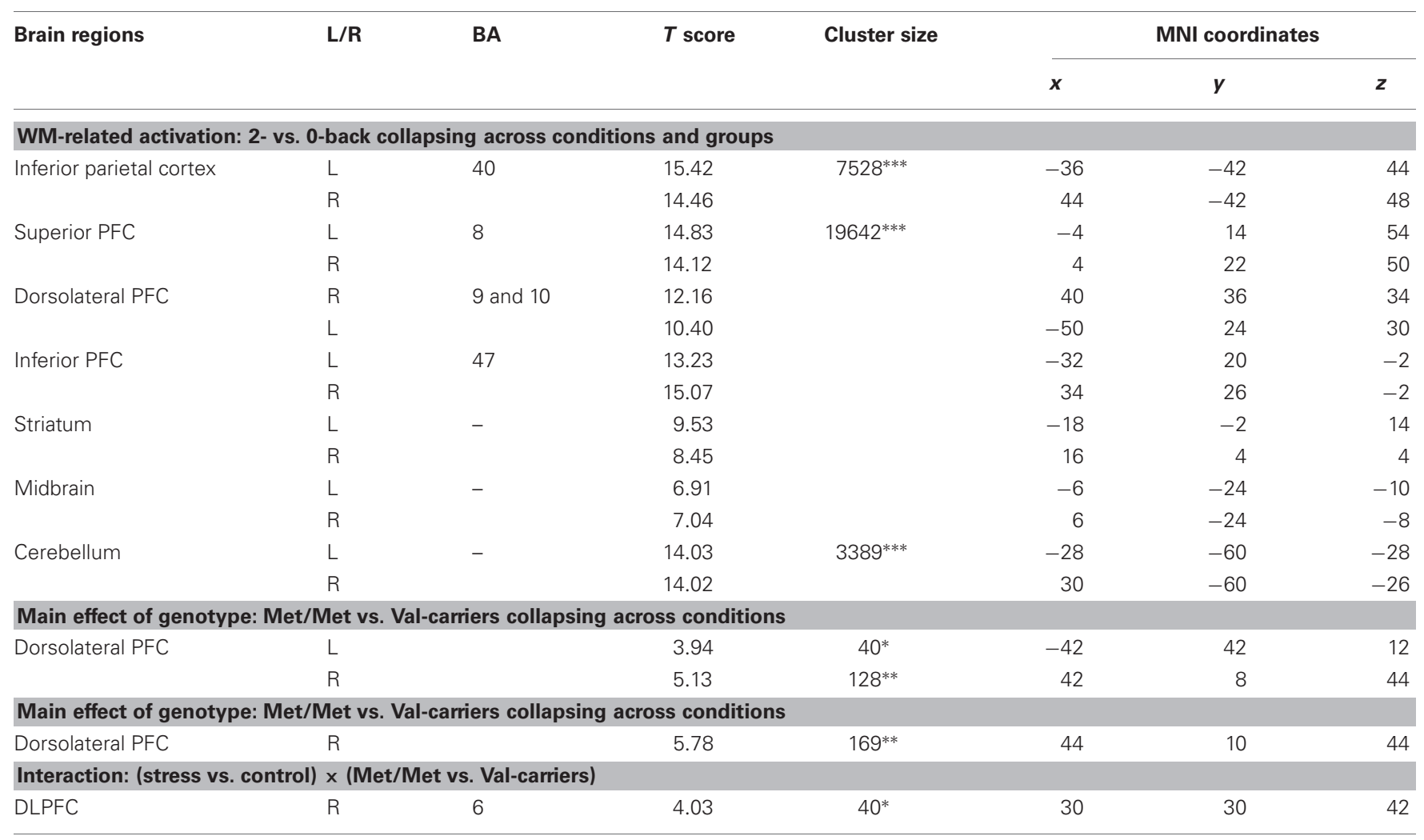

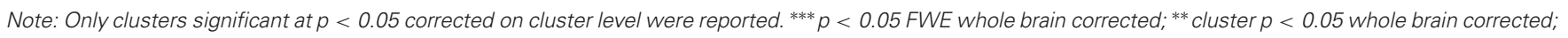

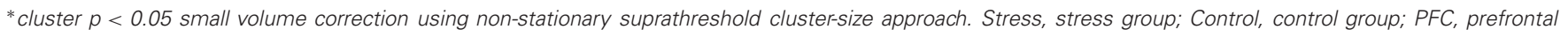
cortex; L, left; $R$, right; BA, Brodmann Area; MNI, MNI coordinates (SPM5). 
Together, these results consistently confirm that the N-back task was indeed embedded in a stressful context in the stress condition.

\section{WM PERFORMANCE}

The mean accuracy and reaction times (RTs) are included in Table 3. WM load effects on accuracy and RTs (i.e., differences between 2- vs. 0-back) were submitted to two separate 2 (stress) by 2 (genotype) ANOVAs. For accuracy, we found a main effect of genotype $\left[F_{(1,36)}=4.95, p<0.03\right]$ with worse for Met-homozygotes in general, and an interaction between genotype and stress $\left[F_{(1,36)}=4.47, p<0.04\right]$ (see Figure 2). Further analyses revealed that COMT Met-homozygotes performed worse than Val-carriers in the stress condition $\left[t_{(37)}=2.85, p<0.01\right]$, while no genotype effect was found in the control condition $\left[t_{(37)}=1.31, p>0.20\right]$. For RTs, neither a main effect of genotype nor an interaction effect was found [all $F$ values $<1]$. To explore potential differences between Val-homozygotes and heterozygotes, we also conducted separately an additional 2-by-3 ANOVA by splitting Val-carriers into heterozygotes and Valhomozygotes, two separate, genotype groups for accuracy and RT data. We found a similar (but weaker) main effect of genotype and an interaction between the factors of genotype and stress. We did not find a significantly larger effect for Val-homozygotes than heterozygotes, likely due to the small sample size. Altogether, these results indicate that there was a larger detrimental effect on accuracy in COMT Met-homozygotes as compared to Val-carriers in the stress condition.

\section{STRESS-BY-COMT GENOTYPE INTERACTION IN WM-RELATED ACTIVATION IN THE DORSOLATERAL PFC}

First, by contrasting 2- with 0-back conditions (collapsing across groups), we confirmed activation of a WM-related frontalparietal network (Table 4), indicating robust main effects of WM load. We also found main effect of genotype on the dorsolateral PFC when collapsing across the stress and control conditions, as well as in the control condition alone (Table 4). More important for the question at issue, we found a suprathreshold cluster in the right dorsolateral PFC (local maxima at [30, 30, 42], $t$ value $=4.03$, cluster $p<0.05$ small volume correction using non-stationary suprathreshold cluster-size approach) for the interactive effect of stress (control vs. stress) and genotype (COMT Met-homozygotes vs. Val-Carriers) on this contrast. There was no reliable activation outside of the PFC in this contrast of stress-by-COMT interaction. As shown in Figure 3, stress induction had opposite effects on WM-related activity in this region depending on genotype. We also analyzed the data by splitting Val-carriers into heterozygotes and Val-homozygotes, two separate groups. This analysis revealed almost an identical main effect of genotype and interaction between stress and genotype in the dorsolateral PFC. Val-homozygotes showed numerically lower activation in the DLPFC in the control condition, and larger stress-induced positive effect as relative to heterozygotes. These effects, however, do not reach the level of significance applied to the analysis otherwise. This lower reliability is likely due to the small sample size of Val-homozygotes in our present study.

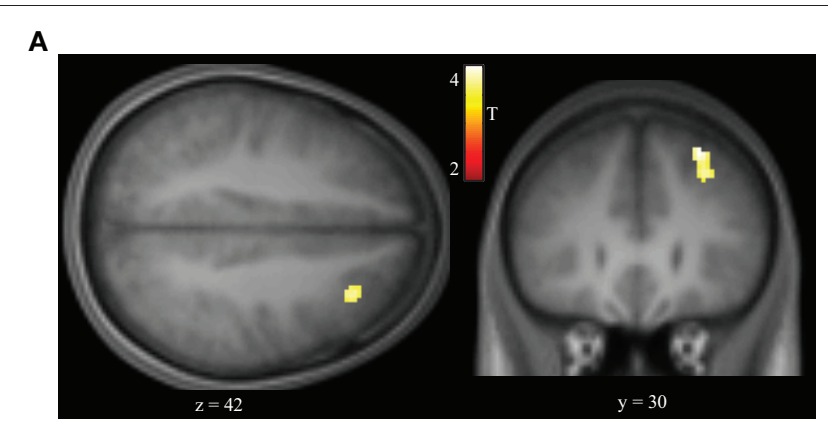

B

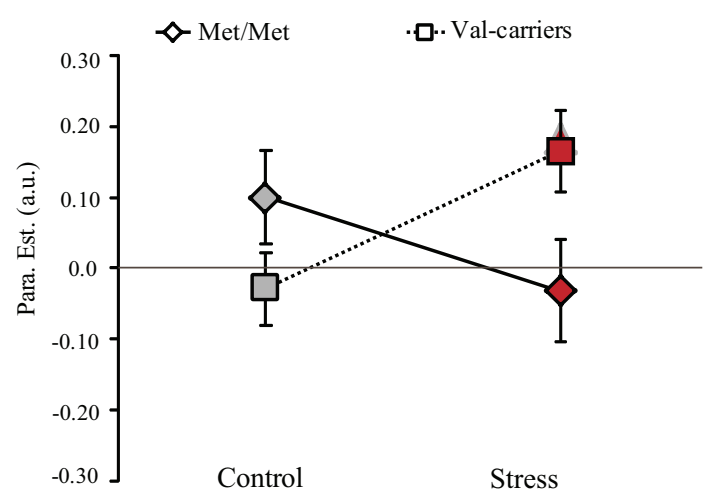

FIGURE 3 | Stress-by-COMT genotype interaction effect on WM-related activity in the dorsolateral PFC. (A) Transversal (left panel) and coronal (right panel) view of activation in the right dorsolateral PFC showing significant stress-by-COMT genotype interaction effect. Statistical parametric maps are superimposed onto spatially normalized and averaged ( $n=39$ ) high-resolution T1-weighted images (thresholded at $p<0.001$ uncorrected for visualization purposes). (B) Bar graphs representing parameter estimates of WM-related activation under control and stress conditions in Met-homozygotes (Met/Met) and Val-carriers. The data for these bar graphs were only extracted to illustrate the interaction effect. Note: Control, control condition; PFC, prefrontal cortex; Stress, stress condition; error bars in the graph represent standard error of mean; T, color coded $t$ values obtained from the whole brain analysis.

\section{STRESS-BY-COMT GENOTYPE INTERACTION IN WM-RELATED DEACTIVATION IN THE MTL}

By contrasting 0- with 2-back conditions (collapsing across groups), we replicated previous findings showing deactivation in regions comprising the "default mode" network (Table 5). For the interactive effect of stress (control vs. stress) and genotype (COMT Met-homozygotes vs. Val-carriers) on this contrast, we found significant clusters in the bilateral anterior MTL extending into amygdala (local maxima at $[-46,2,-20]$ and $[30,0$, $-22]$, both $t$ values $>4.17$, both cluster $p<0.05$ whole-brain corrected). As shown in Figure 4, this interaction exhibits a pattern that is opposite to the one in the dorsolateral PFC- that is, a clear pattern of stress-induced positive and negative effect for Met-homozygotes and Val-carriers, respectively.

\section{DISCUSSION}

The present study investigated whether effects of psychological stress on WM-related neural activity are modulated by a genetic variation in catecholaminergic function (determined by COMT genotype) in humans. As expected, stress induction resulted in 
Table 5 | Brain deactivations related to WM, and modulations of stress and COMT genotype.

\begin{tabular}{|c|c|c|c|c|c|c|c|}
\hline Brain regions & L/R & BA & $T$ score & Cluster size & \multicolumn{3}{|c|}{ MNI coordinates } \\
\hline \multirow[t]{2}{*}{ Posterior cingulate cortex } & - & 31 & 16.05 & $7210^{* * *}$ & 0 & -42 & 36 \\
\hline & $L$ & & 13.49 & & -6 & 54 & 18 \\
\hline Ventral medial PFC & $\mathrm{L}$ & 10 & 12.63 & $5690^{* * *}$ & -4 & 48 & -6 \\
\hline Hippocampus & $\mathrm{R}$ & & 11.29 & $5449 * * *$ & 28 & -20 & -16 \\
\hline \multirow[t]{2}{*}{ Anterior MTL } & $\mathrm{L}$ & $35 / 28$ & 9.81 & & -22 & -10 & -16 \\
\hline & $\mathrm{R}$ & & 6.98 & & 30 & -6 & -18 \\
\hline \multirow[t]{2}{*}{ Insula } & $L$ & 13 & 9.88 & & -36 & -16 & 2 \\
\hline & $\mathrm{R}$ & & 10.52 & & 40 & -16 & 18 \\
\hline \multirow{2}{*}{ Anterior MTL (extending into amygdala) } & $\mathrm{R}$ & - & 4.32 & $103^{* *}$ & 30 & 0 & -22 \\
\hline & & & 4.25 & & 40 & -2 & -24 \\
\hline
\end{tabular}

Note: Only clusters significant at $p<0.05$ corrected on cluster level were reported. ${ }^{* * *} p<0.05$ FWE whole brain corrected; ** cluster $p<0.05$ whole brain corrected. Stress, stress group; Control, control group; MTL, medial temporal lobe; PFC, prefrontal cortex; L, left; R, right; BA, Brodmann Area; MNI, MNI coordinates (SPM5).

COMT genotype-dependent effects on WM performance and WM-related neural activity in the dorsolateral PFC, with a relatively negative impact of stress in COMT Met-homozygotes as opposed to a relatively positive effect in Val-carriers. A similar interaction was found for WM-related deactivation in the anterior MTL, with a stress-induced positive and negative effect for Met-homozygotes and Val-carriers, respectively. As evidenced by elevation of both HR and $\alpha$-amylase, our stress induction procedure succeeded in triggering an increase of sympathetic and noradrenergic activity, and thus resulted in increased central release of stress-sensitive catecholamines (de Kloet et al., 2005; Joels and Baram, 2009; Ulrich-Lai and Herman, 2009), which has been confirmed by our recent pharmacological fMRI study in humans (Hermans et al., 2011). The effectiveness of the stress induction did not differ between genotype groups. We, therefore, discuss how stress-induced elevation of catecholamines, together with genetic variation in catecholaminergic function, accounts for our observed COMT genotype-dependent effects of stress on WM processing.

Our findings of an opposite effect of stress induction in COMT Met-homozygotes as compared to Val-carriers are in line with animal models of the role of catecholamines in the neuromodulation of cognition. Such models assume that catecholaminergic activity exhibits an inverted $\mathrm{U}$-shaped relationship with higherorder prefrontal cognitive function, and that optimal performance is reached at moderate levels of catecholamines (Arnsten and Li, 2005; Aston-Jones and Cohen, 2005; Arnsten, 2007, 2009). Thus, these models predict that the effect of stress-induced elevation of catecholaminergic activity may vary depending on the baseline. In humans, homozygous carriers of the Met allele have lower COMT availability, presumably resulting in higher catecholaminergic activity at baseline. Our findings show that a moderate level of stress has a relatively negative impact on neurocognitive functioning in these individuals, possibly because an elevation of catecholaminergic activity shifts the PFC functioning toward a supraoptimal state at the right side of the alleged inverted-U shaped curve (see Figure 5A). Interestingly, we found an opposite effect in Val-carriers, where a similar amount of stress had a relatively positive effect. To interpret this pattern of results, it is important to consider that the stressor used in this study was relatively mild. For instance, we observed a decrease of cortisol from pre- to post-stressor levels in the control condition but no significant change in the stress condition. Exposure to more stressful situations (i.e., higher levels of cortisol induced) has been found to result generally in detrimental effects on WM processing (Oei et al., 2006; Schoofs et al., 2008, 2009), particularly when high levels of cortisol and noradrenergic activity occurred concurrently (Elzinga and Roelofs, 2005; Schoofs et al., 2008). Moreover, we previously found detrimental effects of acute stress on activity in the dorsolateral PFC across an unselected female sample (Qin et al., 2009), but that study did not implement a crossover design (i.e., repeated testing) and may, therefore, have been slightly more stressful for the participants in the stress condition. It is well possible that higher levels of acute stress would also have a negative impact in COMT Val-carriers. Thus, various (i.e., decreased or improved) effects of acute stress on PFC functions observed in previous studies (Wang et al., 2005; Porcelli et al., 2008; Pruessner et al., 2008; Weerda et al., 2010) may be partly explained by the non-linear relationship between levels of stress-induced catecholamines and prefrontal functioning, and potential interactions with individual differences in basal catecholaminergic availability. In sum, our findings suggest that individuals with high catecholaminergic availability at baseline (COMT Met-homozygotes) are more susceptible to the 


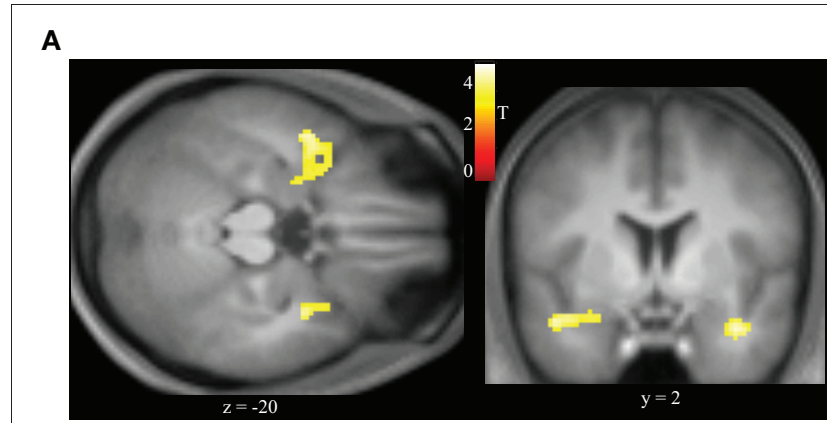

B

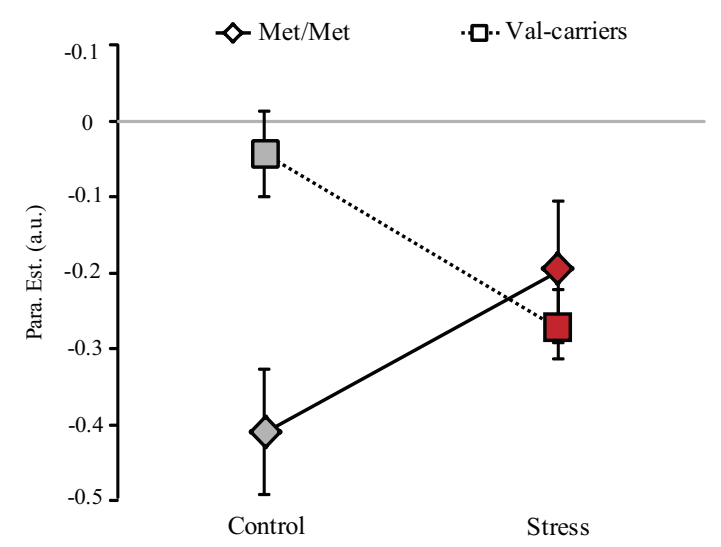

FIGURE 4 | Stress-by-COMT genotype interaction effect on WM-related deactivation in the MTL. (A) Transversal (left panel) and coronal (right panel) view of deactivation in the bilateral anterior MTL showing significant stress-by-COMT genotype interaction effect. Statistical parametric maps are superimposed onto spatially normalized and averaged $(n=39)$ high-resolution T1-weighted images (thresholded at $p<0.001$ uncorrected for visualization purposes). (B) Bar graphs representing parameter estimates of WM-related deactivation under control and stress conditions in Met-homozygotes (Met/Met) and Val-carriers. The data for these bar graphs were extracted illustrating the interaction effect. Note: Control, control condition; MTL, medial temporal lobe; Stress, stress condition; error bars in the graph represent standard error of mean; T, color coded $t$ values obtained from the whole brain analysis.

detrimental effects of stress, whereas Val-carriers appear more resilient.

Animal studies have shown that at a cellular level, the prefrontal WM network is strengthened through actions of a2A-adrenoceptors and increased neural firing via inhibition of cAMP-HCN (cyclic adenosine monophosphate-hyperpolarization-activated cyclic nucleotide-gated cation channel) signaling (Wang et al., 2007), while optimal levels of dopamine decrease task-irrelevant neuronal firing by increasing cAMP-HCN signaling (Arnsten, 2007, 2009; Vijayraghavan et al., 2007). Interestingly, recent studies in behaving primates suggest that elevated levels of catecholamines under stress impair prefrontal functioning by excessive cAMP-HCN and phosphatidylinositol-protein kinase C (PKC) intracellular signaling pathways (Arnsten, 2007, 2009). Activation of these two stress-sensitive signaling pathways is thought to be regulated by molecular inhibitors provided by enzymes such as COMT (Arnsten, 2009). The valine-tomethionine substitution at codon 158 in the COMT protein may

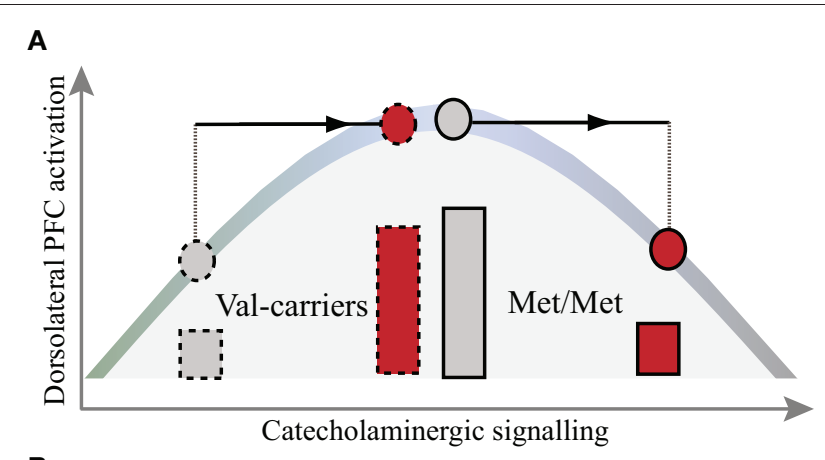

B

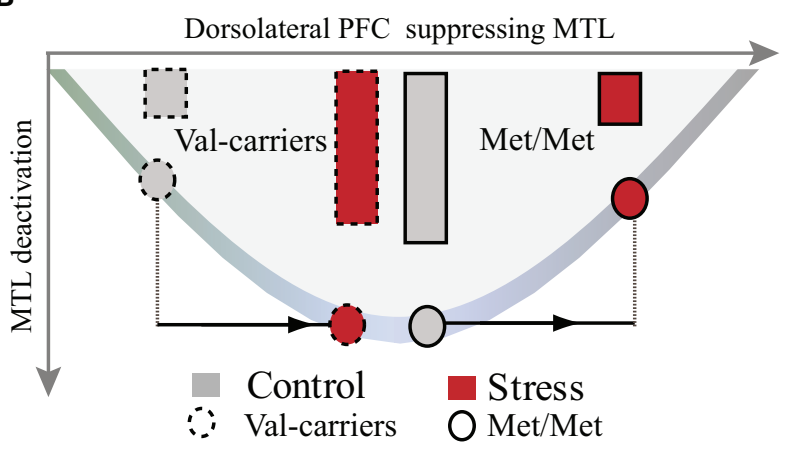

FIGURE 5 | A heuristic model illustrating the effects of COMT genotype and stress on WM-related activation in the dorsolateral PFC and deactivation in the MTL. Stress-by-COMT genotype interaction on the inverted $U$-shaped curve between levels of catecholamines and dorsolateral PFC function (A) and its suppression of MTL function (B). COMT Val-carriers, with presumably lower baseline levels of catecholamines, start from the sub-optimal left end of the inverted U-shaped curve, with suboptimal prefrontal WM function and less MTL suppression. Under moderate psychological stress, elevation of catecholamines may shift catecholaminergic signaling toward an optimal state (strong PFC activation and MTL suppression). In contrast, COMT Met-homozygotes, with high baseline catecholamines, might already start closer to the peak of the curve. Stress-induced elevation of catecholamines may therefore more easily shift these individuals toward a supra-optimal state. Note: The bars represent the magnitude of neural activation in the dorsolateral PFC and deactivation in the MTL, respectively. PFC, prefrontal cortex; MTL, medial temporal lobe.

reduce the ability of COMT to inhibit the activation of these stress-sensitive pathways. By showing a genotype-dependent difference in stress resilience, with relatively stronger stress sensitivity in human carriers of the COMT Met allele, our data provide the first evidence for these notions in humans.

Human neuroimaging studies consistently show that WMrelated activation of the dorsal frontoparietal "executive" network is accompanied by a reciprocal deactivation in regions comprising the "default mode" network (DMN), including the MTL and amygdala (Selemon and Goldman-Rakic, 1988; Dolcos and McCarthy, 2006; Esposito et al., 2006; Arnsten, 2009). Our data robustly replicate these findings, but moreover reveal an interaction between stress and COMT genotype in the bilateral anterior MTL, extending into the amygdala. This interaction shows an opposite pattern to the one observed in the dorsolateral PFC (see Figures 4 and $\mathbf{5 A}, \mathbf{B}$ ), with less deactivation after stress in COMT Met-homozygotes as compared with Val-carriers. 
This finding may be a consequence of the concomitant effect on frontal regions, resulting in altered efficacy of these regions to suppress the DMN. An alternative, but partly complementary, explanation of this finding is that stress-sensitive catecholaminergic changes may exert regionally specific effects on PFC functioning (Aston-Jones and Cohen, 2005; Shin et al., 2005; Dolcos and McCarthy, 2006; Etkin and Wager, 2007), but at the same time have their opposite effects on neural processes in limbic regions, particularly the MTL and the amygdala (Coull et al., 2001; de Kloet et al., 2005; Chamberlain et al., 2006; Admon et al., 2009; van Marle et al., 2009). The function of such a large-scale reallocation of neural resources (Bouret and Sara, 2005; Sara, 2009; Oei et al., 2011) may be to effectuate a hypervigilant brain state promoting rapid behavioral adaptation to adverse conditions at the cost of elaborative executive functions.

It is worth noting that our stress induction procedure refers to an emotion focused, moderate acute psychological stressor rather than socially and cognitively oriented stressors such as the Trier social stress test (TSST) and arithmetic calculation with negative evaluation (Kirschbaum et al., 1993; Dickerson and Kemeny, 2004; Wang et al., 2005; Pruessner et al., 2008). The reason why we did not observe a reliable increase (but nevertheless a significant difference between stress and neutral conditions) in cortisol lies probably in the specifics of the experimental design. The genetic analysis required a within-subject design reducing the contextual novelty effects substantially. Furthermore, we opted for a short succession of the two conditions for reasons of practicality. Nevertheless, converging evidence from multiple elegant readout measurements in the present study as well as our previous studies, including increase in hear rate and cortisol, changes in pupil dilation responses (Henckens et al., 2009; Qin et al., 2012) and increase in $\alpha$-amylase and amygdala perfusion (Cousijn et al., 2010, 2012), firmly promote the conclusion

\section{REFERENCES}

Admon, R., Lubin, G., Stern, O., Rosenberg, K., Sela, L., BenAmi, H., and Hendler, T. (2009). Human vulnerability to stress depends on amygdala's predisposition and hippocampal plasticity. Proc. Natl. Acad. Sci. U.S.A. 106, 14120-14125.

Arnsten, A. F. (2007). Catecholamine and second messenger influences on prefrontal cortical networks of "representational knowledge": a rational bridge between genetics and the symptoms of mental illness. Cereb. Cortex 17(Suppl. 1), i6-i15.

Arnsten, A. F. (2009). Stress signalling pathways that impair prefrontal cortex structure and function. Nat. Rev. Neurosci. 10, 410-422.

Arnsten, A. F., and Goldman-Rakic, P. S. (1998). Noise stress impairs prefrontal cortical cognitive function in monkeys: evidence for a hyperdopaminergic mechanism. Arch. Gen. Psychiatry 55, 362-368.
Arnsten, A. F., and Li, B. M. (2005). Neurobiology of executive functions: catecholamine influences on prefrontal cortical functions. Biol. Psychiatry 57, 1377-1384.

Aston-Jones, G., and Cohen, J. D. (2005). An integrative theory of locus coeruleus-norepinephrine function: adaptive gain and optimal performance. Annu. Rev. Neurosci. 28, 403-450.

Bertolino, A., Caforio, G., Blasi, G., de Candia, M., Latorre, V., Petruzzella, V., Altamura, M., Nappi, G., Papa, S., Callicott, J. H., Mattay, V. S., Bellomo, A., Scarabino, T., Weinberger, D. R., and Nardini, M. (2004). Interaction of COMT (Val(108/158)Met) genotype and olanzapine treatment on prefrontal cortical function in patients with schizophrenia. Am. J. Psychiatry

Bishop, S. J., Fossella, J., Croucher, C. J., and Duncan, J. (2008). COMT val158met genotype affects 161, 1798-1805.

that our emotion focused stress induction procedure was effective. That is, our procedure indeed successfully induced a state characterized by hyperactivation of catecholaminergic systems and negative mood (Arnsten and Goldman-Rakic, 1998; Arnsten, 2009; Hermans et al., 2011; Shackman et al., 2011; Weymar et al., 2011).

Behaviorally, we found COMT genotype-dependent effects of moderate acute psychological stress on WM performance, but no main effect of genotype at baseline in the control condition. The lack of a genotype effect on baseline WM performance may be attributed to a lack of sensitivity at a behavioral level (Meyer-Lindenberg and Weinberger, 2006). A pretraining procedure prior to $\mathrm{AMRI}$ scanning may also play a role in reducing the potential genotype effect. Nonetheless, we found a robust main effect of genotype on WM-related activity in the dorsolateral PFC, indicating that brain imaging genetics may have higher sensitivity for subtle genetic effects on putative neural activity related to cognition and behavior, and thus serving as so-called intermediate phenotype (Meyer-Lindenberg and Weinberger, 2006).

In conclusion, the present study demonstrates COMT genotype-dependent effects of moderate stress on WM performance, WM-related activation of the dorsolateral PFC, and WM-related deactivation in the anterior MTL, with a more negative impact of stress in the genotype that is associated with higher baseline levels of catecholamines. These findings are in line with animal models of catecholaminergic function that assume an inverted U-shaped relationship between catecholaminergic activity and PFC-dependent higher-order cognitive functions.

\section{ACKNOWLEDGMENTS}

This work was supported by the Netherlands Organization for Scientific Research (NWO) (918.66.613, 451.07.019, and 446.10.010).

recruitment of neural mechanisms supporting fluid intelligence. Cereb. Cortex 18, 2132-2140.

Bouret, S., and Sara, S. J. (2005) Network reset: a simplified overarching theory of locus coeruleus noradrenaline function. Trends Neurosci. 28, 574-582.

Brett, M., Anton, J. L., Valabregue, R., and Poline, J. B. (2002). Region of interest analysis using an SPM toolbox. Neuroimage 16, Abstract:497.

Chamberlain, S. R., Muller, U., Blackwell, A. D., Robbins, T. W., and Sahakian, B. J. (2006). Noradrenergic modulation of working memory and emotional memory in humans. Psychopharmacology (Berl) 188, 397-407.

Chen, J., Lipska, B. K., Halim, N., Ma, Q. D., Matsumoto, M., Melhem, S., Kolachana, B. S., Hyde, T. M., Herman, M. M., Apud, J., Egan, M. F., Kleinman, J. E., and Weinberger, D. R. (2004). Functional analysis of genetic variation in catecholO-methyltransferase (COMT): effects on mRNA, protein, and enzyme activity in postmortem human brain. Am. J. Hum. Genet. 75, 807-821.

Coull, J. T., Nobre, A. C., and Frith, C. D. (2001). The noradrenergic alpha2 agonist clonidine modulates behavioural and neuroanatomical correlates of human attentional orienting and alerting. Cereb. Cortex 11, 73-84.

Cousijn, H., Rijpkema, M., Qin, S., van Marle, H. J., Franke, B., Hermans, E. J., van Wingen, G., and Fernandez, G. (2010). Acute stress modulates genotype effects on amygdala processing in humans. Proc. Natl. Acad. Sci. U.S.A. 107, 9867-9872.

Cousijn, H., Rijpkema, M., Qin, S., van Wingen, G. A., and Fernandez, G. (2012). Phasic deactivation of the medial temporal lobe enables working memory processing under stress. Neuroimage 59, 1161-1167. 
de Kloet, E. R., Joels, M., and Holsboer, F. (2005). Stress and the brain: from adaptation to disease. Nat. Rev. Neurosci. 6, 463-475.

Dickerson, S. S., and Kemeny, M. E. (2004). Acute stressors and cortisol responses: a theoretical integration and synthesis of laboratory research. Psychol. Bull. 130, 355-391.

Dolcos, F., and McCarthy, G. (2006). Brain systems mediating cognitive interference by emotional distraction. J. Neurosci. 26, 2072-2079.

Drabant, E. M., Hariri, A. R., MeyerLindenberg, A., Munoz, K. E., Mattay, V. S., Kolachana, B. S., Egan, M. F., and Weinberger, D. R. (2006). Catechol O-methyltransferase val158- met genotype and neural mechanisms related to affective arousal and regulation. Arch. Gen. Psychiatry 63, 1396-1406.

Elzinga, B. M., and Roelofs, K. (2005). Cortisol-induced impairments of working memory require acute sympathetic activation. Behav. Neurosci. 119, 98-103.

Esposito, F., Bertolino, A., Scarabino, T., Latorre, V., Blasi, G., Popolizio, T., Tedeschi, G., Cirillo, S., Goebel, R., and Di Salle, F. (2006). Independent component model of the default-mode brain function: assessing the impact of active thinking. Brain Res. Bull. 70, 263-269.

Etkin, A., and Wager, T. D. (2007). Functional neuroimaging of anxiety: a meta-analysis of emotional processing in PTSD, social anxiety disorder, and specific phobia. Am. J. Psychiatry 164, 1476-1488.

Forman, S. D., Cohen, J. D., Fitzgerald, M., Eddy, W. F., Mintun, M. A., and Noll, D. C. (1995). Improved assessment of significant activation in functional magnetic resonance imaging (fMRI): use of a clustersize threshold. Magn. Reson. Med. 33, 636-647.

Friston, K. J., Holmes, A. P., Worsley, K. J., Poline, J. P., Frith, C. D., and Frackowiak, R. S. J. (1995). Statistical parametric maps in functional imaging: a general linear approach. Hum. Brain Mapp. 2, 189-210.

Goldman-Rakic, P. S. (1995). Architecture of the prefrontal cortex and the central executive. Ann. N.Y. Acad. Sci. 769, 71-83.

Hayasaka, S., Phan, K. L., Liberzon, I., Worsley, K. J., and Nichols, T. E. (2004). Nonstationary clustersize inference with random field and permutation methods. Neuroimage 22, 676-687.

Henckens, M. J., Hermans, E. J., Pu, Z., Joels, M., and Fernandez, G.
(2009). Stressed memories: how acute stress affects memory formation in humans. J. Neurosci. 29, 10111-10119.

Hermans, E. J., van Marle, H. J., Ossewaarde, L., Henckens, M. J., Qin, S., van Kesteren, M. T., Schoots, V. C., Cousijn, H., Rijpkema, M., Oostenveld, R., and Fernandez, G. (2011). Stressrelated noradrenergic activity prompts large-scale neural network reconfiguration. Science 334, 1151-1153.

Hidalgo, V., Villada, C., Almela, M., Espin, L., Gomez-Amor, J., and Salvador, A. (2011). Enhancing effects of acute psychosocial stress on priming of non-declarative memory in healthy young adults. Stress 15, 329-338.

Joels, M., and Baram, T. Z. (2009). The neuro-symphony of stress. Nat. Rev. Neurosci. 10, 459-466.

Kirschbaum, C., Kudielka, B. M. Gaab, J., Schommer, N. C., and Hellhammer, D. H. (1999). Impact of gender, menstrual cycle phase, and oral contraceptives on the activity of the hypothalamuspituitary-adrenal axis. Psychosom. Med. 61, 154-162.

Kirschbaum, C., Pirke, K. M., and Hellhammer, D. H. (1993). The "Trier Social Stress Test"-a tool for investigating psychobiological stress responses in a laboratory setting. Neuropsychobiology 28, 76-81.

Kudielka, B. M., and Kirschbaum, C. (2005). Sex differences in HPA axis responses to stress: a review. Biol. Psychol. 69, 113-132.

Lewis, R. S., Nikolova, A., Chang, D. J., and Weekes, N. Y. (2008). Examination stress and components of working memory. Stress 11, 108-114.

Lotta, T., Vidgren, J., Tilgmann, C., Ulmanen, I., Melen, K., Julkunen, I., and Taskinen, J. (1995). Kinetics of human soluble and membrane-bound catechol O-methyltransferase: a revised mechanism and description of the thermolabile variant of the enzyme. Biochemistry 34, 4202-4210.

Luethi, M., Meier, B., and Sandi, C. (2008). Stress effects on working memory, explicit memory, and implicit memory for neutral and emotional stimuli in healthy men. Front. Behav. Neurosci. 2:5. doi: 10.3389/neuro.08.005.2008

Mannisto, P. T., and Kaakkola, S. (1999). Catechol-O-methyltransferase (COMT): biochemistry, molecular biology, pharmacology, and clinical efficacy of the new selective COMT inhibitors. Pharmacol. Rev. 51, 593-628.

Mason, J. W. (1968). A review of psychoendocrine research on the pituitary-adrenal cortical system. Psychosom. Med. 30, 576-607.

Mattay, V. S., Goldberg, T. E., Fera, F., Hariri, A. R., Tessitore, A., Egan, M. F., Kolachana, B., Callicott, J. H., and Weinberger, D. R. (2003). Catechol O-methyltransferase val158-met genotype and individual variation in the brain response to amphetamine. Proc. Natl. Acad. Sci. U.S.A. 100, 6186-6191.

McEwen, B. S. (2007). Physiology and neurobiology of stress and adaptation: central role of the brain. Physiol. Rev. 87, 873-904.

Meyer-Lindenberg, A., Nichols, T., Callicott, J. H., Ding, J., Kolachana, B., Buckholtz, J., Mattay, V. S., Egan, M., and Weinberger, D. R. (2006). Impact of complex genetic variation in COMT on human brain function. Mol. Psychiatry 11, 797, 867-877.

Meyer-Lindenberg, A., and Weinberger, D. R. (2006). Intermediate phenotypes and genetic mechanisms of psychiatric disorders. Nat. Rev. Neurosci. 7, 818-827.

Mobbs, D., Petrovic, P., Marchant, J. L., Hassabis, D., Weiskopf, N., Seymour, B., Dolan, R. J., and Frith, C. D. (2007). When fear is near: threat imminence elicits prefrontal-periaqueductal gray shifts in humans. Science 317, 1079-1083.

Nichols, T., and Hayasaka, S. (2003). Controlling the familywise error rate in functional neuroimaging: a comparative review. Stat. Methods Med. Res. 12, 419-446.

Oei, N. Y., Everaerd, W. T., Elzinga, B. M., van Well, S., and Bermond, B. (2006). Psychosocial stress impairs working memory at high loads: an association with cortisol levels and memory retrieval. Stress 9, 133-141.

Oei, N. Y., Veer, I. M., Wolf, O. T., Spinhoven, P., Rombouts, S. A., and Elzinga, B. M. (2011). Stress shifts brain activation towards ventral "affective" areas during emotional distraction. Soc. Cogn. Affect. Neurosci. 7, 403-412.

Papaleo, F., Crawley, J. N., Song, J., Lipska, B. K., Pickel, J., Weinberger, D. R., and Chen, J. (2008). Genetic dissection of the role of catechol-Omethyltransferase in cognition and stress reactivity in mice. J. Neurosci. 28, 8709-8723.

Porcelli, A. J., Cruz, D., Wenberg, K., Patterson, M. D., Biswal, B. B., and Rypma, B. (2008). The effects of acute stress on human prefrontal working memory systems. Physiol. Behav. 95, 282-289.

Pruessner, J. C., Dedovic, K., KhaliliMahani, N., Engert, V., Pruessner, M., Buss, C., Renwick, R., Dagher, A., Meaney, M. J., and Lupien, S. (2008). Deactivation of the limbic system during acute psychosocial stress: evidence from positron emission tomography and functional magnetic resonance imaging studies. Biol. Psychiatry 63, 234-240.

Qin, S., Hermans, E. J., van Marle, H. J., and Fernández, G. (2012). Understanding low reliability of memories for neutral information encoded under stress: alterations in memory-related activation in the hippocampus and midbrain. J. Neurosci. 32, 4032-4041.

Qin, S., Hermans, E. J., van Marle, H. J., Luo, J., and Fernandez, G. (2009). Acute psychological stress reduces working memory-related activity in the dorsolateral prefrontal cortex. Biol. Psychiatry 66, 25-32.

Sara, S. J. (2009). The locus coeruleus and noradrenergic modulation of cognition. Nat. Rev. Neurosci. 10, 211-223.

Schoofs, D., Preuss, D., and Wolf, O. T. (2008). Psychosocial stress induces working memory impairments in an n-back paradigm. Psychoneuroendocrinology 33, 643-653.

Schoofs, D., Wolf, O. T., and Smeets, T. (2009). Cold pressor stress impairs performance on working memory tasks requiring executive functions in healthy young men. Behav. Neurosci. 123, 1066-1075.

Selemon, L. D., and Goldman-Rakic, P. S. (1988). Common cortical and subcortical targets of the dorsolateral prefrontal and posterior parietal cortices in the rhesus monkey: evidence for a distributed neural network subserving spatially guided behavior. J. Neurosci. 8, 4049-4068.

Shackman, A. J., Maxwell, J. S., McMenamin, B. W., Greischar, L. L., and Davidson, R. J. (2011). Stress potentiates early and attenuates late stages of visual processing. J. Neurosci. 31, 1156-1161.

Shin, L. M., Wright, C. I., Cannistraro, P. A., Wedig, M. M., McMullin, K., Martis, B., Macklin, M. L., Lasko, N. B., Cavanagh, S. R., Krangel, T. S., Orr, S. P., Pitman, R. K., Whalen, P. J., and Rauch, S. L. (2005). A functional magnetic resonance imaging study of amygdala and medial prefrontal cortex responses to overtly presented fearful faces in posttraumatic stress disorder. Arch. Gen. Psychiatry 62, 273-281.

Strahler, J., Berndt, C., Kirschbaum, C., and Rohleder, N. (2010). Aging 
diurnal rhythms and chronic stress: distinct alteration of diurnal rhythmicity of salivary alpha-amylase and cortisol. Biol. Psychol. 84, 248-256.

Ulrich-Lai, Y. M., and Herman, J. P. (2009). Neural regulation of endocrine and autonomic stress responses. Nat. Rev. Neurosci. 10, 397-409.

van Marle, H. J., Hermans, E. J., Qin, S., and Fernandez, G. (2009). From specificity to sensitivity: how acute stress affects amygdala processing of biologically salient stimuli. Biol. Psychiatry 66, 649-655.

Vijayraghavan, S., Wang, M. Birnbaum, S. G., Williams, G. V., and Arnsten, A. F. (2007). Inverted-U dopamine D1 receptor actions on prefrontal neurons engaged in working memory. Nat. Neurosci. 10, 376-384.

Wang, J., Rao, H., Wetmore, G. S., Furlan, P. M., Korczykowski, M., Dinges, D. F., and Detre, J. A.
(2005). Perfusion functional MRI reveals cerebral blood flow pattern under psychological stress. Proc. Natl. Acad. Sci. U.S.A. 102, 17804-17809.

Wang, M., Ramos, B. P., Paspalas, C. D., Shu, Y., Simen, A., Duque, A., Vijayraghavan, S., Brennan, A., Dudley, A., Nou, E., Mazer, J. A., McCormick, D. A., and Arnsten, A. F. (2007). Alpha2A-adrenoceptors strengthen working memory networks by inhibiting cAMP-HCN channel signaling in prefrontal cortex. Cell 129, 397-410.

Ward, B. D. (2000). Simultaneous Inference for FMRI Data. AFNI 3dDeconvolve Documentation. Medical College of Wisconsin. Watson, D., Clark, L. A., and Tellegen, A. (1988). Development and validation of brief measures of positive and negative affect: the PANAS scales. J. Pers. Soc. Psychol. 54, 1063-1070.
Weerda, R., Muehlhan, M., Wolf, O. T., and Thiel, C. M (2010). Effects of acute psychosocial stress on working memory related brain activity in men. Hum. Brain Mapp. 31, 1418-1429.

Weymar, M., Schwabe, L., Low, A., and Hamm, A. O. (2011). Stress sensitizes the brain: increased processing of unpleasant pictures after exposure to acute stress. J. Cogn Neurosci. [Epub ahead of print].

Worsley, K. J., Marrett, S., Neelin, P., Vandal, A. C., Friston, K. J., and Evans, A. C. (1996). A unified statistical approach for determining significant signals in images of cerebral activation. Hum. Brain Mapp. 4, 58-73.

Conflict of Interest Statement: The authors declare that the research was conducted in the absence of any commercial or financial relationships that could be construed as a potential conflict of interest.

Received: 30 November 2011; accepted: 07 April 2012; published online: 11 May 2012.

Citation: Qin S, Cousijn H, Rijpkema $M$, Luo J, Franke B, Hermans EJ and Fernández $G$ (2012) The effect of moderate acute psychological stress on working memory-related neural activity is modulated by a genetic variation in catecholaminergic function in humans. Front. Integr. Neurosci. 6:16. doi: 10.3389/fnint.2012.00016

Copyright (c) 2012 Qin, Cousijn, Rijpkema, Luo, Franke, Hermans and Fernández. This is an open-access article distributed under the terms of the Creative Commons Attribution Non Commercial License, which permits noncommercial use, distribution, and reproduction in other forums, provided the original authors and source are credited. 